\title{
Inference of Temperature and Density Profiles via Forward Modeling of an $X$-ray Imaging Crystal Spectrometer within the Minerva Bayesian Analysis Framework
}

\author{
A. Langenberg, ${ }^{1,}$ a) J. Svensson, ${ }^{1}$ O. Marchuk, ${ }^{2}$ G. Fuchert, ${ }^{1}$ S. Bozhenkov, ${ }^{1} H_{\text {. Damm, }}{ }^{1}$ E. Pasch,,${ }^{1}$ A. Pavone, ${ }^{1}$ \\ H. Thomsen, ${ }^{1}$ N.A. Pablant, ${ }^{3}$ R. Burhenn, ${ }^{1}$ R.C. Wolf, ${ }^{1}$ and the W7-X Teamb) \\ 1) Max-Planck-Institut für Plasmaphysik, 17491 Greifswald, Germany \\ ${ }^{2)}$ Forschungszentrum Jülich GmbH, Institut für Energie- und Klimaforschung - Plasmaphysik, 52425 Jülich, \\ Germany \\ 3) Princeton Plasma Physics Laboratory, Princeton, NJ, USA
}

(Dated: 29 May 2019)

At the Wendelstein 7-X stellarator, the X-ray imaging crystal spectrometer provides line integrated measurements of ion and electron temperatures, plasma flows, as well as impurity densities from a spectroscopic analysis of tracer impurity radiation. In order to infer the actual profiles from line integrated data, a forward modeling approach has been developed within the Minerva Bayesian analysis framework. In this framework, the inversion is realized on the basis of a complete forward model of the diagnostic, including error propagation and utilizing Gaussian processes for generation and inference of arbitrary shaped plasma parameter profiles. For modeling of line integrated data as measured by the detector, the installation geometry of the spectrometer, imaging properties of the crystal, and Gaussian detection noise are considered. The inversion of line integrated data is achieved using the maximum posterior method for plasma parameter profile inference and a Markov chain Monte Carlo sampling of the posterior distribution for calculating uncertainties of the inference process. The inversion method shows a correct and reliable inference of temperature and impurity density profiles from synthesized data within the estimated uncertainties along the whole plasma radius. The application to measured data yields a good match of derived electron temperature profiles to data of the Thomson scattering diagnostic for central electron temperatures between $2-5 \mathrm{keV}$ using argon impurities.

PACS numbers: Valid PACS appear here

\section{INTRODUCTION}

For the measurement of radial profiles of basic plasma parameters, like ion and electron temperature $T_{i}, T_{e}$, poloidal flow velocity $v_{\phi}$ and impurity density $n_{Z}$, an X-ray imaging crystal spectrometer (XICS) has been brought into operation at the Wendelstein 7-X (W7$\mathrm{X})$ stellarator ${ }^{1} \mathbb{4}$. In such spectrometers of the Johann type $e^{5}$, wavelength selection is achieved with a spherically bent crystal, creating a $2 \mathrm{D}$ intensity pattern on a pixilated detector with energy dispersion in horizontal direction and spatial resolution in vertical direction. The spectral resolution $\lambda / \Delta \lambda \approx 9900$ of XICS enables a resolution of the line position and the line width of impurity line radiation with an accuracy of $5 \times 10^{-5} \AA$. Spectra recorded at different vertical positions across the detector correspond to different lines of sight of the spectrometer. Therefore, crystal spectrometers provide line integrated measurements of plasma parameters 6 -11. The lines of sight are located in a bean shaped poloidal plasma cross section of W7-X. From the line integrated spectra, information about the plasma parameters can be deduced. In some cases, these line integrated measurements are a good approximation of the actual plasma parameter

\footnotetext{
a) Electronic mail: andreas.langenberg@ipp.mpg.de

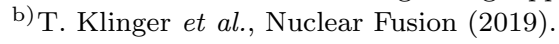

profiles $7 / 12[13$. However, in general significant discrepancies between line integrated measurements and the actual profiles may occur ${ }^{1415}$, especially in non-circular plasma cross-sections like the ones in the W7-X stellarator ${ }^{2}$. For axial symmetric plasmas, the inversion can be done using Abel inversion 16 19. In the W7-X stellarator, the plasma cross-sections are not circular. Thus, more sophisticated inversion methods are required 20, 27. Some general challenges in unfolding line integrated spectral information are: A: Performing a simultaneous tomographic inversion of all plasma parameter profiles having direct impact on measured spectra. B: Finding the most likely inversion profiles including a comprehensive treatment of measurement uncertainties. C: Utilizing an adequate parametrization of unknown plasma parameter profiles. This paper presents an inversion method, based on a forward modeling of XICS (section II) within the Minerva Bayesian Analysis framework ${ }^{28}$. As will be shown, the framework provides an inversion of arbitrary shaped plasma parameter profiles for arbitrary plasma cross section shapes (section III A and B), including a comprehensive treatment of uncertainties (section III C). In section IV, results of inversion for non standard plasmas will be discussed, followed by a $T_{e}$ profile validation of actually measured data (section V).

An additional motivation using Bayesian analysis is a joint data analysis of multiple diagnostics, as several diagnostics share identical observations, e.g. $T_{e}$ profiles measured by the electron cyclotron emission diagnostic ${ }^{29}$, 
the Thomson scattering diagnostic ${ }^{30}$, and $\mathrm{XICS}^{1}$.

\section{MINERVA BAYESIAN ANALYSIS FRAMEWORK}

\section{A. Bayesian Analysis}

Bayesian Analysis has become a standard statistics method for the inversion of line integrated measurements in plasma physics and is utilized for data analysis of several plasma diagnostics 28131 35. In this approach, predicted data $\boldsymbol{D}^{*}$, depending on a set of model parameters $\boldsymbol{N}$ through a physics model $f(\boldsymbol{N})=\boldsymbol{D}^{*}$, are related to observed data $\boldsymbol{D}$ in terms of a Probability Density Function, considering the measurement uncertainties, e.g. measurement noise.

The likelihood distribution $\boldsymbol{P}(\boldsymbol{D} \mid \boldsymbol{N})$ denotes the probability of observed data for given parameters $\boldsymbol{N}$. Applied to an imaging spectrometer measuring observed data $\boldsymbol{D}$, the likelihood of observed counts for all pixels of a pixilated detector is given by

$$
\boldsymbol{P}(\boldsymbol{D} \mid \boldsymbol{N})=\prod_{p=0}^{N_{p i x}-1} \frac{1}{\sqrt{2 \pi} \sigma_{p}} \exp \left(-\frac{\left(\boldsymbol{D}_{p}^{*}-\boldsymbol{D}_{p}\right)^{2}}{2 \sigma_{p}^{2}}\right) .
$$

Here, a normal distribution with a standard deviation $\sigma_{p}$ is assumed for the photon statistics ${ }^{36}$. The predicted and observed numbers of counts are $\boldsymbol{D}_{p}^{*}$ and $\boldsymbol{D}_{\boldsymbol{p}}$ for each pixel $\boldsymbol{p}$. The solution to the inversion problem in this formulation is the posterior distribution over the model parameters $\boldsymbol{N}$, which is given by Bayes formula

$$
\boldsymbol{P}(\boldsymbol{N} \mid \boldsymbol{D})=\boldsymbol{P}(\boldsymbol{D} \mid \boldsymbol{N}) \boldsymbol{P}(\boldsymbol{N}) / \boldsymbol{P}(\boldsymbol{D})
$$

yielding a probability distribution of the free parameters, given the measured data. The prior distribution $\boldsymbol{P}(\boldsymbol{N})$ reflects any knowledge on the model parameters before the measurement took place, $\boldsymbol{P}(\boldsymbol{D})$ is a normalization constant. The priors used in this Bayesian analysis approach are defined by stationary Gaussian processes, used for the regularization of plasma parameter profiles (see next paragraph). Here, informative priors, namely truncated normal distributions were used. Those are normal distributions centered at zero with widths of $50 \mathrm{keV}$ and $10^{17} \mathrm{~m}^{-3}$, respectively, and of zero values for negative temperatures or densities. This way, a prior knowledge of non-physical negative as well as unexpected high values of the free parameters has been considered.

\section{B. Forward Modeling}

The dependencies between model parameters and modeled data are defined within a Bayesian graphical model inside the utilized Minerva Bayesian analysis framework 28 . Plasma parameters entering the model as free model parameters $\boldsymbol{N}$ are radial profiles of ion and

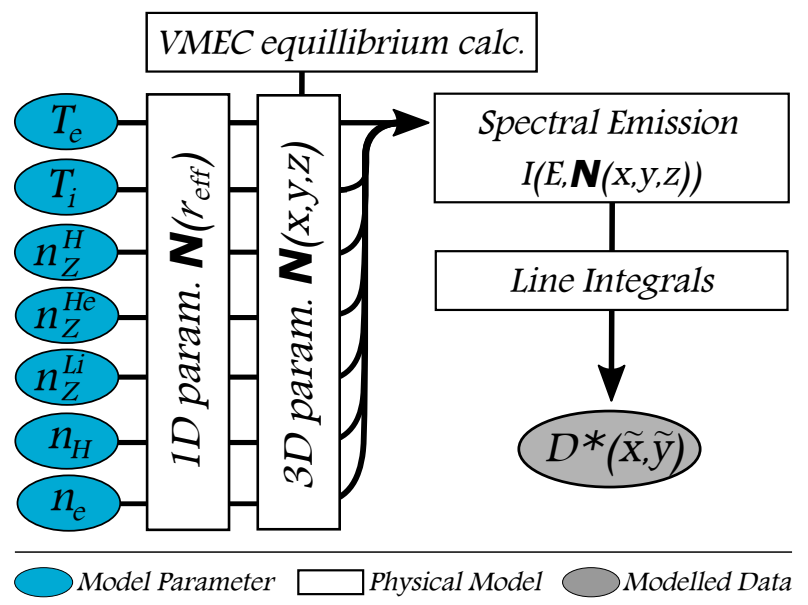

FIG. 1. Simplified scheme for the forward model of an Xray imaging spectrometer in the Minerva Bayesian analysis framework.

electron temperatures $T_{i}$ and $T_{e}$, electron density $n_{e}$, different ionization stages (hydrogen-, helium-, and lithiumlike) of impurity densities $n_{Z}^{H / H e / L i}$, and the neutral hydrogen density $n_{H}$. Due to their high spectral resolution, imaging spectrometers cover just a narrow wavelength range that, dependent on the crystal choice, usually enables the observation of only one impurity species in three to maximum four neighboring charge states.

A simplified scheme for the forward model of an X-ray imaging spectrometer is shown in Fig 1. Model parameters (blue nodes), e.g. profiles of $T_{e}, T_{i}, \ldots n_{e}$, are parameterized as 1D functions of the plasma radius $\boldsymbol{N}(\rho)$ using an equally spaced grid of 15 points along $\rho$ with linear interpolation between points. Here, $\rho=\sqrt{\psi / \psi_{L C F S}}$ is defined as the square root of the poloidal magnetic flux $\psi$, normalized to the magnetic flux of the last closed flux surface $\psi_{L C F S}$. In order to achieve a high flexibility in the inference of plasma parameter profiles, stationary Gaussian processes are utilized for the regularization of inverted profiles 37 . Here, a prior knowledge on the stiffness of profiles is set with the squared exponential covariance function

$$
\operatorname{cov}\left(\rho_{i}, \rho_{j}\right)=\sigma_{f}^{2} \exp \left(-\frac{\left(\rho_{i}-\rho_{j}\right)^{2}}{2 \sigma_{r}^{2}}\right)
$$

defining the covariance between any two positions $\rho_{i}$ and $\rho_{j}$ of the plasma radius with a length scale $\sigma_{r}$ and amplitude parameter $\sigma_{f}$. Profiles of low stiffness that are expected to vary on a small length scale with high amplitude can be described properly by choosing $\sigma_{r} \ll 1$ and $\sigma_{f} \gg 1$, whereas for profiles of high stiffness, $\sigma_{r}$ and $\sigma_{f}$ would be set to $\sigma_{r}=\sigma_{f} \approx 1$. In this paper, $\sigma_{r}$ and $\sigma_{f}$ have been set to $\sigma_{r}=0.1-0.3$ and $\sigma_{f}=10$ as a compromise between sufficient flexibility to describe arbitrary shaped profiles (see Fig 7 a) and a minimized computational time. Ideally, $\sigma_{r}$ and $\sigma_{f}$ should be optimized using a Bayesian Occam's razor approach, which is done for linear problems in $\frac{37}{\text {. Th }}$ This problem is how- 
ever highly nonlinear and such optimization is outside the scope of the current work. For a more detailed discussion, the reader is referred to standard literature on Gaussian processes $37 / 38$. In order to meet the symmetry constraint of a zero gradient at the plasma axis for the 15 grid points of plasma parameter profiles as well as the interpolated values between the grid points next to the plasma center, a two step approach has been followed: First, a virtual zero slope observation at $\rho=0$ has been included in the model, as proposed and discussed in detail in $\frac{38}{3}$. Second, for the two grid points next to the plasma center at $\rho=0$ and $\rho=0.07$, the linear interpolation has been replaced by a spline fit, fulfilling the zero gradient approach.

Compared to other common representations as e.g. basis function approaches 39140 , Gaussian processes combines parametrization and regularization of profiles and also adjusts the model complexity through just one entity, the length scale parameter ${ }^{22 / 38 / 41]}$. The model can be upgraded (see e.g. Li et al. $!^{22}$ ) by allowing non stationary Gaussian processes, taking into account different length scales across the plasma radius. For the present temperature and density profile inference, this is however not required.

The 1D profiles $\boldsymbol{N}(\rho)$ are mapped to the 3D geometry $\boldsymbol{N}(x, y, z)$ using equilibrium flux surfaces $\psi(x, y, z)$ as given by calculations done with the variational moments equilibrium code (VMEC) ${ }^{42}$, with spatial coordinates $x, y$, and $z$. The spectral emission is evaluated along lineof-sight integrals considering the designed installation geometry of the spectrometer, modeling a 2D energy and spatial resolved intensity pattern $D(\tilde{x}, \tilde{y})$ (gray node) at the position of the detector, with the pixel coordinates $\tilde{x}$ and $\tilde{y}$ on the detector. The individual, actually cone shaped lines of sight, having a maximal vertical extent of $2 \mathrm{~cm}$, have been approximated by line integrals using the sum over spectral emissions at 100 equidistant positions along each line of sight. Plasma parameters $\left(T_{e}\right.$, $T_{i}, n_{e}$ etc.) are all assumed to be flux surface functions, as is the emitted radiation. Possible poloidal or toroidal asymmetries are neglected in this model.

Atomic calculations of rate coefficients for the corre-

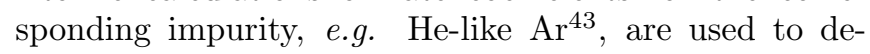
termine the spectral emission for the local plasma parameter set. Here, the following atomic processes have been considered 44: A. Excitation of the He-like ground state, B. dielectronic recombination, C. recombination of H-like ions, D. inner-shell excitation and ionization, and E. charge exchange recombination with neutral background gas hydrogen. All of the direct excitation lines $(w, x, y, z)$, all dielectronic lines up to $n=4$, and all inner shell excitation lines were included in the final spectrum. For reference, Fig 2 shows contributions of the different atomic processes to the modeled spectrum and their dependencies on different Ar charge states. A detailed discussion including rate coefficients can be found in the work of Marchuk $\frac{43}{4}$. The shapes of spectral lines have been modeled as Voigt profiles, resulting from a
Lorentzian contribution from the lifetime of lines, a Gaussian contribution from Doppler broadening, and the instrumental profile.

One should add that the systematic errors in the atomic data affect the determination of electron temperature $T_{e}$ and the concentration of impurity charge stages, but not the ion temperature $T_{i}$ and flow velocity profiles. In the former case, $T_{e}$ and $n_{Z}$ are derived from measured line intensity ratios and absolute line intensities in direct comparison to theoretically expected line ratios and intensities $\underline{43}$. For instance, $T_{e}$ mainly affects the measured line ratio of the main excitation $w$ line and the dielectronic $k$ satellite line or dielectronic lines of the $n=3$ group, see Fig2. Thus, errors in the theoretic excitation and dielectronic rate coefficients would have direct impact on the inferred $T_{e}$ values. The independent Rmatrix and Coulomb-Born calculations for the excitation rate coefficients of the $w, x, y$, and $z$ lines demonstrate a deviation on the order of $3-5 \% 43$. The comparison for the intensities of dielectronic satellites was performed in the work of Bitter et al $\frac{45}{45}$ and error bars in the new MZ data 46 , AUTOSTRUCTURE ${ }^{47}$, or FAC code ${ }^{48}$ calculations of the intensity of the satellites for the group of $n=2$ and $n=3$ is expected to be within $5-10 \%$. Therefore, the impact of the different set of atomic data for determination of inverted $T_{e}$ and $n_{Z}$ profiles is expected to be nonnegligible and foreseen to be investigated systematically in the near future. However, a comparison of experimentally derived XICS $T_{e}$ profiles using presently available atomic data with $T_{e}$ measurements of other diagnostics shows a fairly good agreement ${ }^{49}$ as also discussed in section V (see Fig, 8) of this work.

\section{Inference of Plasma Parameter Profiles from Line Integrated Data}

The implemented forward model enables the inference of model parameters, given measured data. Variation of the model parameters with respect to an optimal match between forward modeled and measured data yields the model parameters, similar to a common least squares fit. One of the key features of Bayesian Analysis used for inversion of plasma parameter profiles is to infer not just one single, but a representative set of all possible plasma parameter profiles - given the model assumptions, yielding the measured spectra within the experimental uncertainties. For this purpose, the maximum posterior (MAP) method and the Markov chain Monte Carlo (MCMC) sampling $\sqrt{50} \sqrt{52}$ are utilized together.

The aim of the MAP inference is to determine one set of model parameters yielding a best fit to measured data. Since the posterior $\boldsymbol{P}(\boldsymbol{N} \mid \boldsymbol{D})$ measures the agreement between modeled and measured data, cf. Eq. (1), the MAP method maximizes the value of the posterior by variation of the model parameters $\boldsymbol{N}$.

In order to quantify uncertainties of the inversion process, a direct MCMC sampling of the posterior distribution 


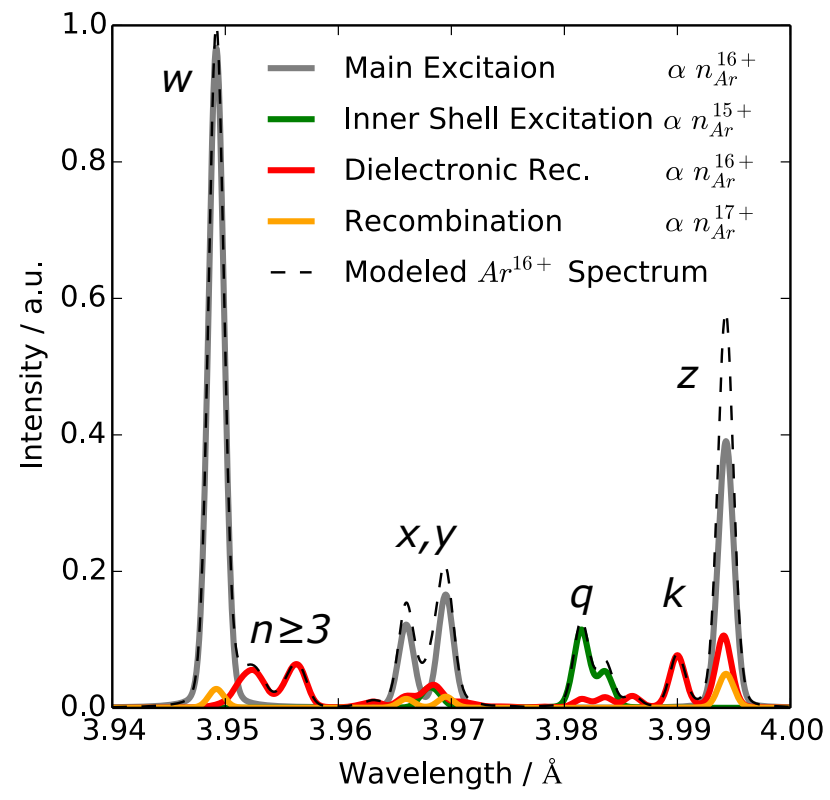

FIG. 2. Modeled $A r^{16+}$ spectrum (dashed line), its decomposition into the different atomic processes (colored, solid lines) and their dependencies on adjacent Ar charge states (legend) for assumed plasma parameters of $T_{e}=T_{i}=1.0 \mathrm{keV}$ and $n_{A r}^{15+/ 16+/ 17+}=1.0 / 5.0 / 0.5 \times 10^{15} \mathrm{~m}^{-3}$.

$\boldsymbol{P}(\boldsymbol{N} \mid \boldsymbol{D})$ using a Metropolis-Hastings algorithm $m^{5153}$ is performed on an $m$-dimensional surface which dimensionality is determined by the number of free model parameters $m$. In this case, $m=75$ according to a set of 5 plasma parameter profiles ( $n_{e}$ and $n_{H}$ kept constant, see next paragraph), each containing $n=15$ points along $\rho$. The precision of an inferred model parameter, like $T_{e}$ of a given flux surface, is determined from the distribution of samples of the Markov chain. Therefore, sampling of the $m$-dimensional posterior distribution yields a scatter of free parameters from which the uncertainties of the free parameters, and so the uncertainty of inferred profiles can be calculated.

Since the electron density can not be inferred from the measured spectra due to the ambiguity of $n_{e}$ with the impurity densities $n_{Z}$, it has not been varied and assumed to be known from other diagnostics. Also the neutral hydrogen background profile $n_{H}$ has not been inverted but estimated based on a diffusion model (section III A). Uncertainties of both profiles are neglected and would in case of consideration give rise to an additional systematic uncertainty for the inferred impurity density profiles.

\section{RESULTS AND DISCUSSION}

\section{A. Model Inputs and Synthesized Data}

In the following, temperature and density profiles used to create noisy data will be referred to as assumed profiles and synthesized data. These synthesized data serve as measured test data for inference of plasma parameter profiles using the Minerva Bayesian analysis framework. Hence, comparing assumed with inferred profiles yields an accurate test of the inversion method.

Fig. 3 a) shows typical profiles used for the forward modeling: Temperature and density profiles, $T_{i}(\rho), T_{e}(\rho)$, $n_{e}(\rho)$, and $n_{Z}^{H / H e / L i}(\rho)$, have been chosen to mimic typically measured profiles at $\mathrm{W} 7-\mathrm{X}^{49154}$, where impurity density profiles are derived from theoretic calculations, using the one dimensional (flux surface averaged) transport code STRAHL ${ }^{55}$. Details on the performed STRAHL code calculations can be found in references 55156 . Since up to now the absolute value of neutrals at the plasma edge of W7-X has not been reported yet, it has been set to an arbitrary, reasonable value of $5 \times 10^{13} \mathrm{~m}^{-3}$. The actual $n_{H}$ profile shape has then been estimated in a one dimensional diffusion model based on the continuity equation

$$
D \frac{\delta n_{H}}{\delta x}=\int_{0}^{x}\left(-n_{e} n_{H} k_{i}+n_{e} n_{i} k_{r}\right) d x^{\prime}
$$

with the diffusion coefficient $D$ defined as

$$
D=\frac{2}{3} \frac{T_{i}}{n_{i} m_{i} k_{c x}} .
$$

Here, $k_{i}, k_{r}$, and $k_{c x}$ are the rate coefficients for ionization, recombination, and charge exchange of hydrogen 57 , $n_{i}$ and $m_{i}$ are the ion density and mass of hydrogen and $r$ is the effective plasma radius $r=a \rho$ with the minor plasma radius $a=0.51 \mathrm{~m}$ as defined by the position of the last closed flux surface. Using the $T_{i}, T_{e}$, and $n_{e}$ profiles of Fig 3 a) in the diffusion model yields the exponential decaying $n_{H}$ profile, also shown in Fig 3 a). From the plasma edge towards the plasma center, the neutral hydrogen density drops by a factor of about 100 . The authors are aware that the utilized 1D model might suffer from inaccuracies especially close to the last closed flux surface and should be optimized using e.g. EMC3Eirene code calculations ${ }^{58}$ when evaluating actual measured data.

All above given profiles have direct impact on the intensities of modeled spectral emission lines. Magnetic flux surfaces were derived using a VMEC solution for the equilibrium of several standard W7-X plasma configurations including the expected plasma pressure profiles. An example of vacuum equilibrium flux surfaces for $\rho=0.1-1.0$ is shown in Fig 3 b).

The resulting modeled $2 \mathrm{D}$ intensity pattern at the position of the pixilated detector is shown in Fig.3 c). According to the utilized detector of XICS (Pilatus 300K, 

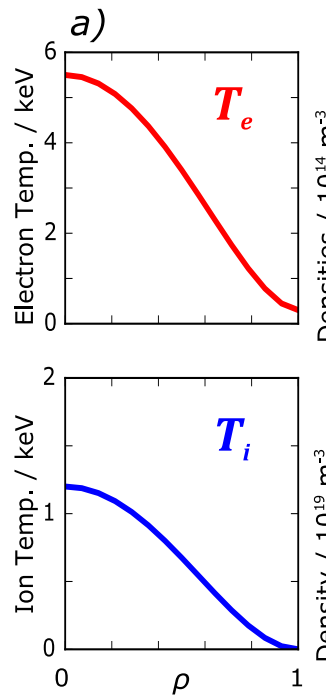

b)

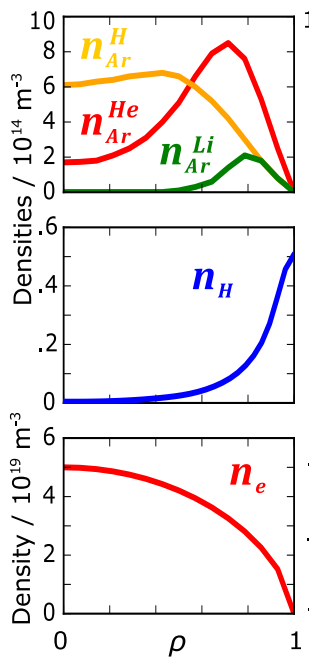

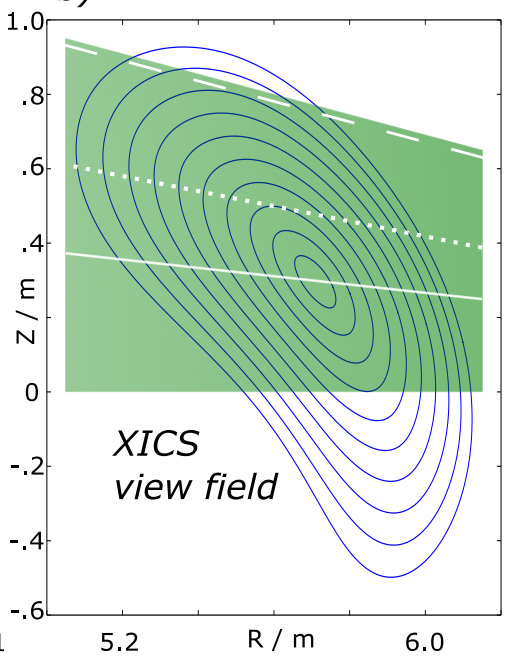

d)

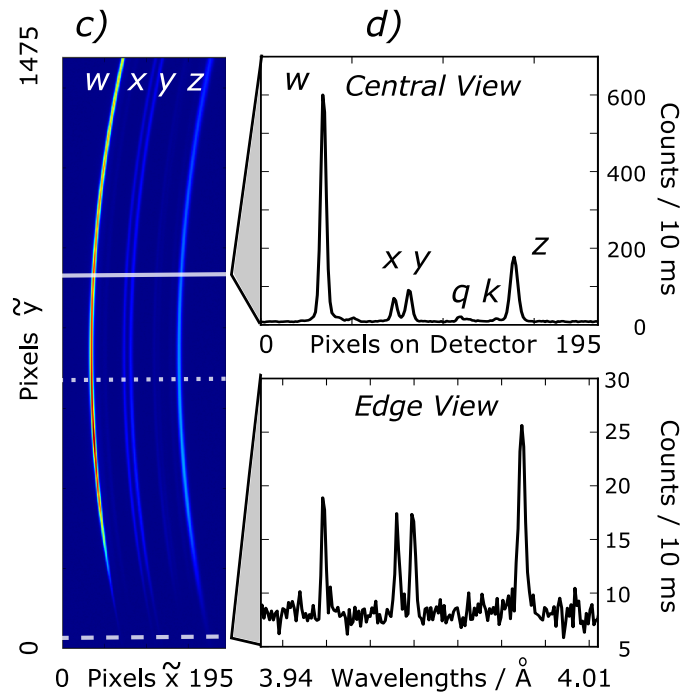

FIG. 3. Forward modeling of the XICS diagnostic for spectral emission of He-like Ar: a) Assumed temperature and density profiles. b) Magnetic flux surfaces derived by VMEC calculations and viewing geometry of XICS in the poloidal cross section of the measurement. c) Modeled 2D intensity pattern on the detector. White solid, dotted, and dashed lines correspond to central, intermediate and edge lines of sight. The uppermost line of sight (edge view) corresponds to the lowest horizontal pixel array (dotted line) and vice versa. d) Synthesized spectra along the central and outermost line of sight with main intense $w, x, y$, and $z$ emission lines and satellites $q$ and $k$.
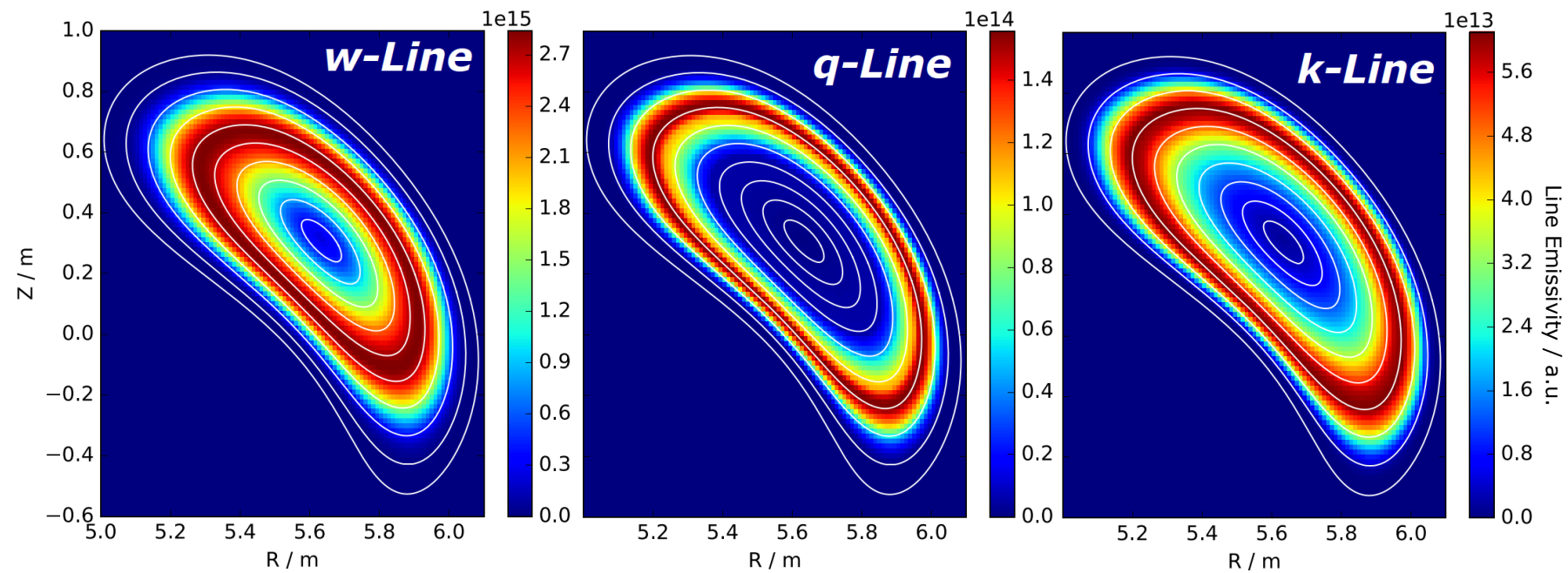

FIG. 4. Synthesized emissivity distribution contours of selected emission lines from the $A r^{16+}$ spectrum, using temperature and density profiles as shown in Fig 3 a).

Dectris Inc., Switzerland), a total number of 1475 x 195 pixels with $172 \times 172 \mu \mathrm{m}^{2}$ pixel size has been modeled. Since the imaging properties of the crystal have been included in the forward model, the focal lines on the detector are curved.

In a poloidal plane at the toroidal position of XICS lines of sight, magnetic flux surfaces are "bean shaped". As a consequence of this asymmetry, the $2 \mathrm{D}$ intensity pattern on the detector is also asymmetric with respect to the central line of sight, (straight line in Fig $3 \mathrm{~b}+\mathrm{c}$ ). In fact, the line of sight with peaked intensity of the $w$ line (dotted line in Fig $3 \mathrm{c}$ ) does not correspond to the central line of sight, in contrast to observations usually made in axially symmetric plasmas. Thus, the geometry of the setup requires a proper modeling of sight line contributions for a correct inversion result.

To illustrate the changes in the spectra, moving from the plasma center towards the plasma edge, line integrated spectra along a single central and an edge line of sight are shown in Fig 3 d). The radially outward decreasing ion temperature results in narrower linewidths towards the edge, while the decreasing electron temperature changes 
the line ratios and reduces the overall intensity of spectral lines (Fig $3 \mathrm{~d}$ d). In order to simulate a realistic diagnostic measurement situation in a stellarator plasma environment, a typical statistical noise corresponding to a signal to noise ratio (SNR) of 150 for the central and 10 for the edge line of sight has been added to the data ${ }^{2}$. The absolute intensities of modeled spectra in Fig 3 d) were matched to measured intensities observed at a pulsed $\mathrm{Ar}$ gas puff injecting $N_{A r} \approx 2 \times 10^{17}$ atoms whereas the gas puff of main $\mathrm{H}$ atoms was on the order of $N_{H} \approx 1 \times 10^{21}$ atoms. This corresponds to a detected signal of $6 \times 10^{4}$ counts/s for the $w$ line intensity along the central line of $\operatorname{sight} \sqrt{56}$.

Fig 4 shows the radial distribution of synthesized emissivities for selected emission lines, namely the $w, q$, and $k$ lines. As the $w$ and the $k$ line intensities are proportional to the $n_{A r}^{16+}$ density, their emissions follow basically the assumed $n_{A r}^{16+}$ profile with a peaking around $\rho=0.6-$ 0.8 and low emission at the plasma center. The slightly changed peaking position of the emissivities result from the additional temperature dependence being different for the $w$ and $k$ lines. The $q$ line emissivity is narrower and zero in the plasma center according to the narrow $n_{A r}^{15+}$ profile compared to the profile of $n_{A r}^{16+}$. Note, that according to Fig 2, the $w, q$, and $z$ lines are affected by several different atomic processes and as a result, also depend on more than on density profile as e.g. the $q$ and $z$ lines.

\section{B. Inference of Plasma Parameter Profiles}

The inference of plasma parameter profiles is done in two steps: In a first step, the MAP method is used to infer one set of plasma parameter profiles, resembling the synthesized data. In a second step, the posterior distribution is sampled using the MCMC method in order to identify a range of other sets of plasma parameter profiles that would also match the same synthesized data, taking into account the assumed experimental uncertainties. This approach yields a comprehensive treatment of uncertainties related to the inversion process. The inferred profiles have been derived from the mean of the MCMC samples.

For the inversion, a representative number of 20 lines of sight, distributed evenly across the spectrometer's view field, have been modeled. As initial guess profiles for the MAP inference, an arbitrary set of constant temperature and density profiles has been chosen. After 300-500 iterations (depending on the noise level of synthesized data), the posterior converges close to its maximum value. Since each temperature and density profile (except $n_{e}$ ) contributes differently to the various spectral emission lines (see Fig 2), all profiles can be inferred independently from each other given that photon statistics of the spectrum enable a reasonable fit of all line intensities.
Note, that for every iteration the complete forward modeling procedure is performed. In particular, the whole set of spectral emission lines in the observed energy interval $\left(\approx 150\right.$ lines ${ }^{43}$ ) can be modeled and fitted simultaneously to the experimental data. Using this technique, the spectral fitting takes into account the photon statistics $\sigma_{p}$ of each pixel via Eq. (1).

In Fig.5 a) and b), inferred electron and ion temperature profiles $T_{e}(\rho)$ and $T_{i}(\rho)$ (dashed lines) are plotted together with the previously assumed ones (bold solid lines). For comparison, also the averaged $T_{e}$ and $T_{i}$ values integrated along the line of sight path are shown as dots. As can be seen, line integrated temperature values are systematically underestimated, already for standard plasma scenarios whereas the inferred temperature profiles (dashed lines) resemble the assumed ones within the determined uncertainties (gray solid lines). In fact, there is an excellent agreement between inferred and assumed profiles all along the plasma radius with a maximum deviation of less than $100 \mathrm{eV}$. Estimated uncertainties are in the order of $\pm 100 \mathrm{eV}$ for $\rho=0.4-0.9$ and gradually increase towards the plasma center and at the plasma edge.

In Fig 5 c)-e), assumed and inferred Ar density profiles of different charge states are shown. Similar to the situation observed for the temperature profiles, inferred density profiles match the assumed ones within the estimated uncertainties. Moreover, there is a good agreement between assumed and inferred $\mathrm{He}-, \mathrm{Li}-$, and $\mathrm{H}$-like $\mathrm{Ar}$ densities in the region of $\rho=0.1-0.8$ with deviations accompanied by larger uncertainties towards the plasma edge.

\section{Uncertainties of Inferred Profiles}

The gradually increasing uncertainties towards the plasma center and at the plasma edge (solid gray lines in Fig 5p of inferred temperature and density profiles are determined by two basic effects. These are on the one hand a low signal to noise ratio of measured data (for the edge region), and on the other hand the hollow shape of the $n_{A r}^{16+}$ profile accompanied by a low sensitivity of measured data to variations of a plasma parameter on a specific flux surface (like $T_{e}$ or $T_{i}$ close to the plasma center).

A high noise level near the plasma edge is usually caused by low values of electron temperature and electron density (see Fig $3 \mathrm{a}+\mathrm{d}$ ) in combination with a reduced path length of lines of sight crossing the plasma edge region. The reduced impact of core plasma parameters on the measured spectrum is associated with the nature of line integrated measurements: Along a central line of sight, a strong integrated signal of non central spectral emission is superimposed to a comparably weak signal of central emission. This effect is enhanced by the hollow $n_{A r}^{16+}$ density profile. Therefore, deviations between inferred and assumed profiles close to the plasma center and at the plasma edge do cause just marginal differences between 

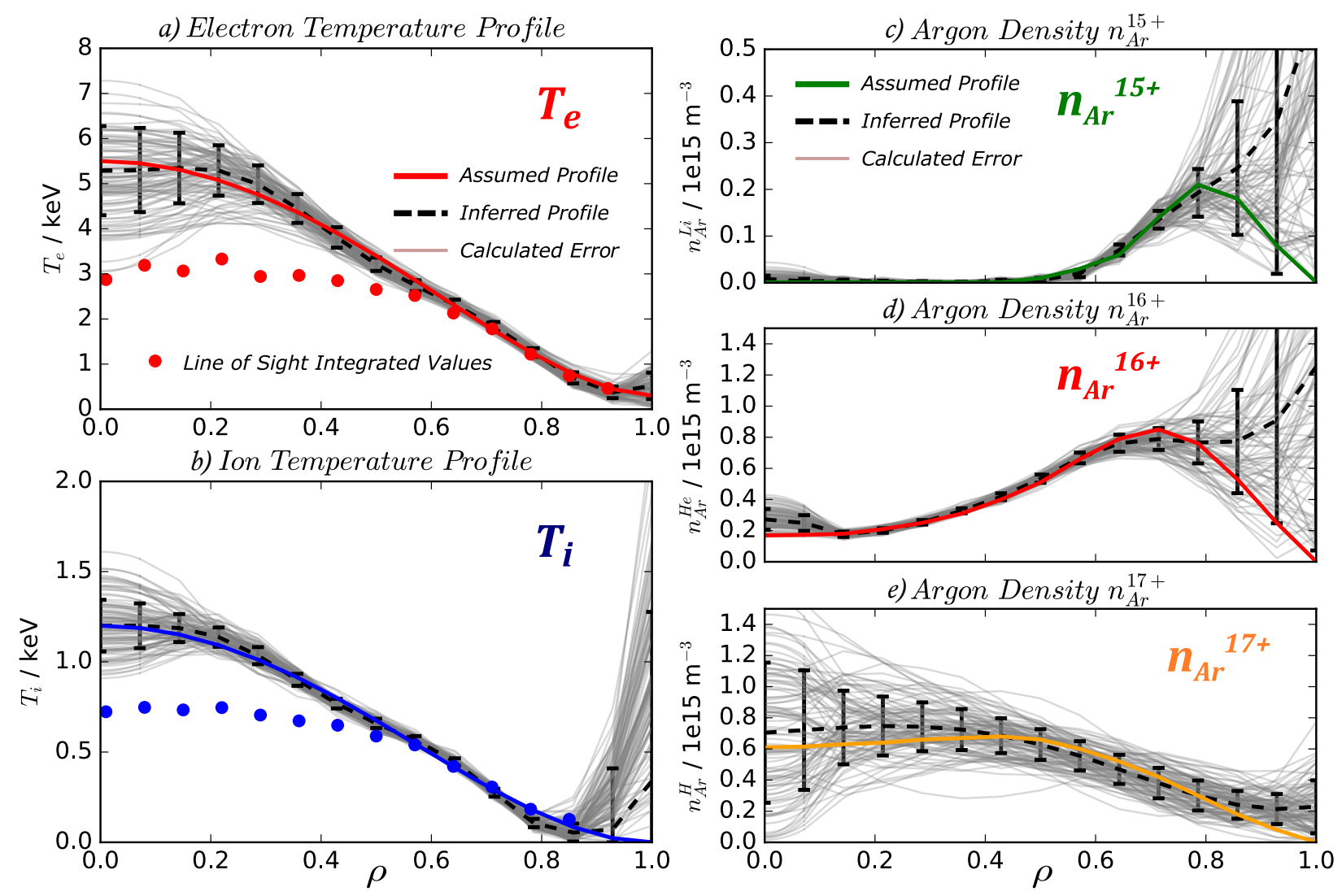

FIG. 5. Left: Assumed (solid lines) and inferred electron and ion temperature profiles (dashed black lines) from synthesized noisy data of XICS. Gray solid lines represent samples from the error distribution indicating the uncertainty profile, error bars illustrate one standard deviation of the sampled profiles. Dots represent electron and ion temperature values, averaged along the XICS lines of sight. Right: Assumed (solid lines) and inferred argon density profiles of different ionization states (dashed black lines) from synthesized noisy data of XICS.

$\log P(\boldsymbol{N} \mid \boldsymbol{D})$

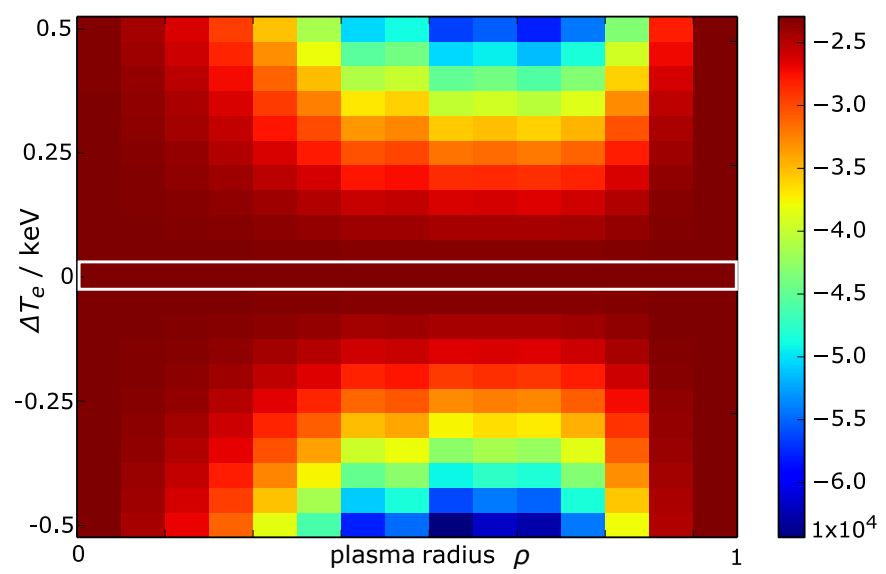

FIG. 6. Two dimensional scan of the posterior with respect to the electron temperature along the plasma radius $\rho$. The white box indicates the maximum values of the posterior.

measured and modeled data.

The uncertainties of a particular temperature or density profile are additionally affected by a rather complex in- terplay of competitive emission line intensities, used for the inversion of distinct profiles. For instance, the gradually increasing uncertainties of the $T_{e}$ profile from $\rho=0.5$ towards the plasma center are driven by the hollow $n_{A r}^{16+}$ density profile that reduces the intensity of spectral emission lines around the plasma center with respect to non central plasma emission from $\rho>0.5$.

The above discussed uncertainties of inferred profiles have been derived by sampling the posterior probability distribution with respect to the model parameters, using MCMC sampling 51. As initial guess parameters, the set of model parameters determined by the MAP method has been used.

For illustration, Fig 6 shows a representative scan of the 2D distribution of the posterior $\boldsymbol{P}\left(\boldsymbol{T}_{\boldsymbol{e}}, \boldsymbol{\rho} \mid \boldsymbol{D}\right)$, varying $T_{e}$ along $\rho$. If $T_{e}$ values are set identical to those used to generate synthesized data $\left(\Delta T_{e}=0\right)$, there is a close match between modeled and synthesized data. The white box in Fig 6 marks the maximum value of the posterior. Any deviation from the assumed $T_{e}$ profile $\left(\Delta T_{e} \neq 0\right.$ in Fig 6$)$ induces a worse match of modeled and synthesized data and thereby also reduces the value of the posterior. Obviously, a variation of $T_{e}$ has different impacts on the pos- 
terior at different magnetic flux surfaces: At the plasma center and at the edge region ( $\rho \approx 0$ and 1 , respectively), the posterior is rather constant when varying $T_{e}$, reflecting a minor contribution of $T_{e}$ on these specific flux surfaces to the synthesized spectrum. In reverse, this causes enhanced uncertainties for the inference of central and edge $T_{e}$ values. At other radial positions $(\rho=0.3-0.8)$, the posterior changes significantly with $T_{e}$, yielding small uncertainties for the inference process. For simplicity, correlations within the $T_{e}$ profile have been neglected for the posterior scan of Fig 6 .

The results of 100 MCMC samples of the posterior distribution for all model parameters (except $n_{e}$ and $n_{H}$ ) are shown in Fig 5 and 7 as gray solid lines. The shown error bars are one standard deviation of sampled profiles. Inferred and assumed temperature and density profiles match within the experimental uncertainties with enhanced uncertainties close to the plasma center and at the plasma edge due to reasons discussed above.

\section{BEYOND STANDARD PLASMA SCENARIOS}

In section III, the inference of temperature and density profiles of a standard plasma scenario as observed during the first operational phase of $\mathrm{W} 7-\mathrm{X}^{[56]}$ has been shown. In this section, the inversion method will be applied to more advanced scenarios like a strongly modulated $T_{e}$ profile, impurity and electron density holes, and high noise levels of measured data. If not indicated otherwise, assumed temperature and density profiles and the noise level of synthesized data have been set equal to those shown in Fig 3 a) and d).

The inference of a fast varying $T_{e}$ profile including a high steepness in the plasma center, a high amplitude of modulation up to $4 \mathrm{keV}$ and 4 turning points is shown in Fig 7 a). Although these kind of $T_{e}$ profiles are quite unexpected, the inversion of such a profile demonstrates the capabilities of the underlying Gaussian processes for the inference of in fact arbitrary shaped profiles. As can be seen, the inferred profile matches the assumed one very well with above discussed deviations and enhanced uncertainties in the plasma center and edge. In particular, the inference does not suffer from a potential oversmoothing of profiles. Inferred $T_{i}$ and density profiles are very similar to the assumed profiles (which are the same as for the standard case shown in Fig 5 b)-e), thus neither the inferred profiles, nor the estimated uncertainties are affected significantly by the strong modulation of the assumed temperature profile. All inferred temperature and density profiles resemble the assumed ones within the estimated uncertainties. The slightly enhanced peaking of the inferred $T_{e}$ profile at $\rho=0.4$ is caused by the so far non optimized choice of the $\sigma_{f}$ and $\sigma_{r}$ parameters, as discussed in section II B of this work.

Although not observed within the past operational phases of W7-X, in large stellarators, highly peaked $T_{e}$ profiles combined with hollow $n_{e}$ profiles as shown in
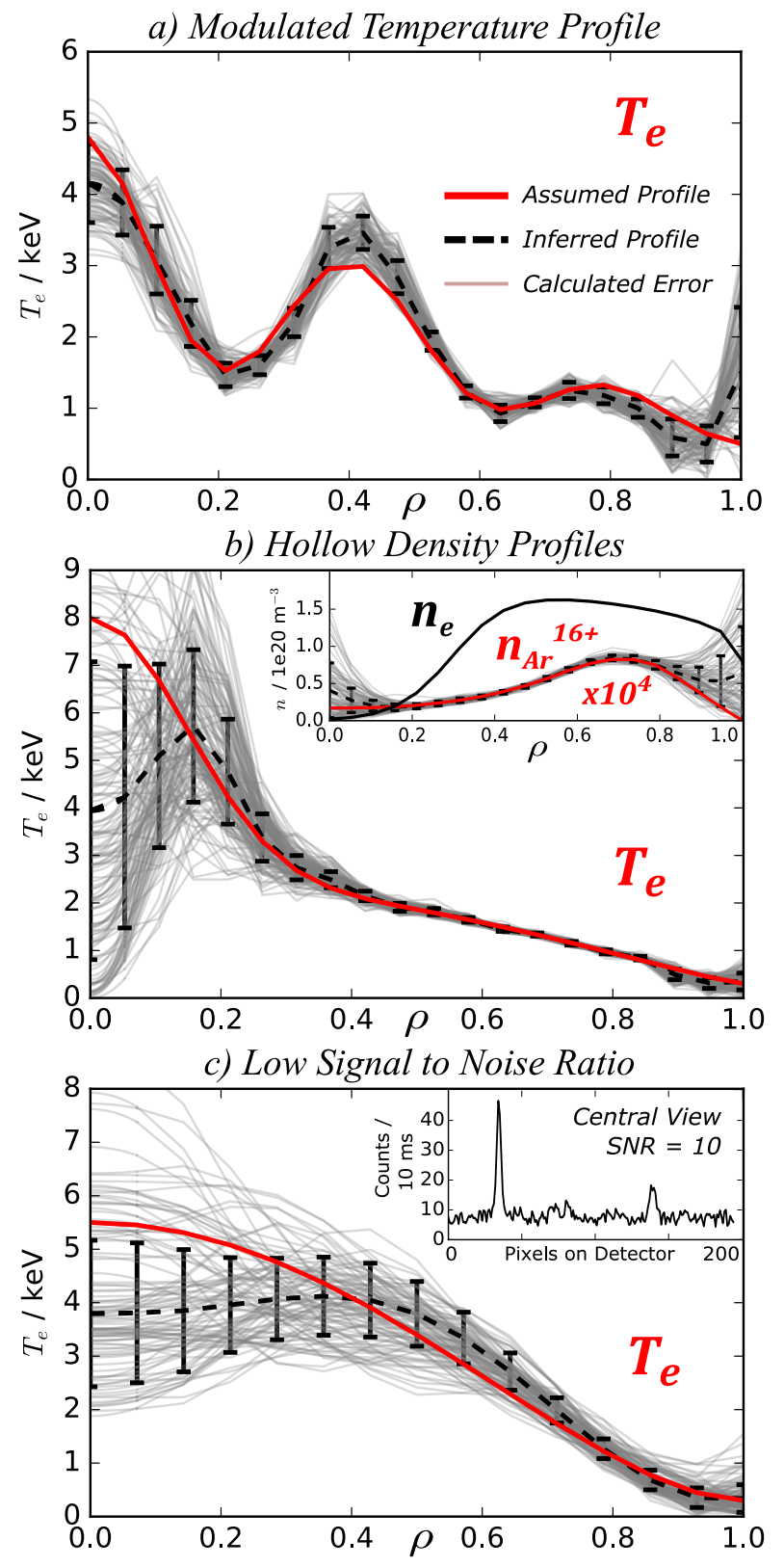

FIG. 7. Inference of $T_{e}$ and $n_{A r}^{H e}$ profiles for non standard plasma scenarios: a) Arbitrary shaped $T_{e}$ profile. b) Impurity and electron density hole. c) Low signal to noise ratio of synthesized data.

Fig.7 b) are predicted in case of non optimized particle refilling rates $\$$. As a consequence of central electron temperatures of $T_{e}>6 \mathrm{keV}$, the central He-like argon density will be ionized, creating an additional impurity hole. The combined electron density and argon impurity density hole can be considered as a worst case scenario for the inference of plasma parameter profiles from Xray imaging spectrometers. Since both parameters contribute linearly to the spectral emission intensity, central electron and impurity densities being close to zero causes the central spectral emission to vanish. Therefore, mea- 
sured data contain little information about the central emission. This fact is reflected in the non convergence of inferred $T_{e}$ and $n_{A r}^{H e}$ profiles towards the assumed ones close to the plasma center. The same is true for the not shown $T_{i}$ and density profiles. However, with recovery of a sufficiently high spectral emission signal for $\rho>0.2$, the convergence of inferred to assumed profiles is restored. The significant non convergence at the plasma center is indicated by the MCMC sampling, yielding significantly larger uncertainties in this region so that inferred and assumed profiles match within the estimated uncertainties along the whole plasma radius.

For all inversions presented so far, a typical signal to noise ratio of measured data $(\mathrm{SNR}=150$ along central line of $\operatorname{sigh} \mathrm{t}^{2}$ ) has been assumed in order to show the achievable precision of the inversion method. Nonetheless, it's also possible to infer profiles from spectra with low signal to noise ratios, as shown in Fig 7 c). Here, measured data with more than one order of magnitude less signal levels $(\mathrm{SNR}=10$ along central line of sight, see inset of Fig 7 c) have been synthesized. As expected, high noise in the spectra induces a worse match between assumed and inferred profiles and increases the averaged estimated uncertainties by a factor of 2 . However, the inferred $T_{e}$ profile still matches the assumed one within the estimated uncertainties. Again, the same is true for inferred $T_{i}$ and density profiles.

The forward model can be upgraded easily to include further physical effects. For instance, an additional model parameter tracing a possible line shift induced by plasma rotation can be added. While toroidal plasma rotation is expected to be small in stellarators, the poloidal rotation induced by radial neoclassical $E_{r}$ fields causes detectable line shifts $\sqrt{60}$ that have been neglected in this work. Additionally, a possible variation of the impurity density on the magnetic flux surface, as expected for highly charged ions 61 , 63, or a possible misalignment of the spectrometer lines of sight can be taken into account. It should be mentioned that results from the first operational phases of W7-X does not show any significant up down asymmetries in the line intensities, different to previous observations made on ALCATOR-C Mod $\sqrt{64}$, but in agreement to theoretical data $\sqrt{65}$. An investigation of this effect for higher $Z$ impurities such as iron or nickel is planned for the future.

\section{EXPERIMENTAL PROFILE VALIDATION}

The presented inversion method has been used successfully on actual measured data for device commissioning purposes $^{1 / 2}$ as well as physics studies $56 \mid 66$. In general, inferred electron temperature profiles show a good match to results of other diagnostics like ECE measurements or Thomson scattering data ${ }^{49}$. For illustration, Fig 8 compares experimentally obtained $T_{e}$ profiles from XICS together with local $T_{e}$ measurements of the Thomson scattering diagnostic ${ }^{30}$ for central electron temperatures be-

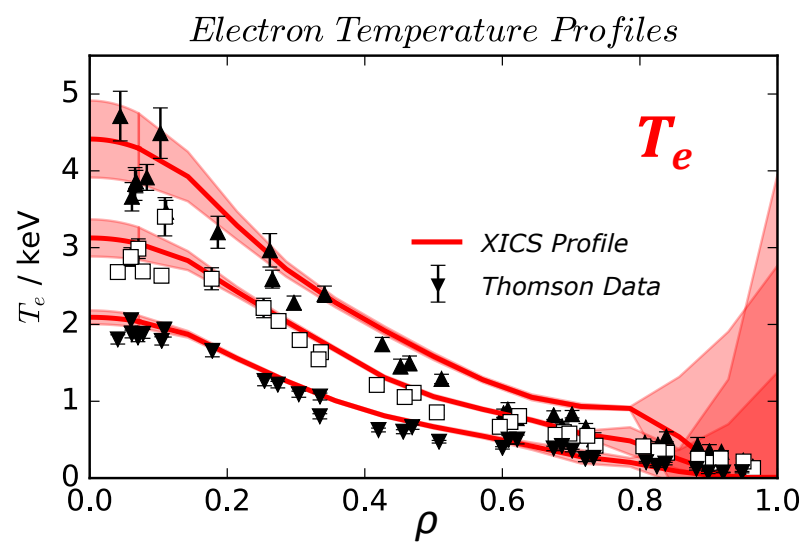

FIG. 8. Comparison of experimentally obtained $T_{e}$ profiles from the XICS (lines) and Thomson scattering (symbols) diagnostics for three different experiment scenarios of W7-X, including uncertainties (error bars and shaded areas). The corresponding program numbers, line integrated plasma densities, and ECR heating powers were:

$180919.055 @ 1.2 \mathrm{~s}, n_{e}=6.1 \times 10^{19} \mathrm{~m}^{-2}, P_{E C R H}=4.0 \mathrm{MW}$,

(triangles up),

180927.046@3.0 s, $n_{e}=7.9 \times 10^{19} \mathrm{~m}^{-2}, P_{E C R H}=3.4 \mathrm{MW}$, (squares),

180927.046@5.0 s, $n_{e}=7.9 \times 10^{19} \mathrm{~m}^{-2}, P_{E C R H}=1.8 \mathrm{MW}$, (triangles down).

tween $T_{e}=2-5 \mathrm{keV}$. As evident from Fig 8 , there is a reasonably good match of XICS $T_{e}$ profiles and the local $T_{e}$ measurements of the Thomson scattering diagnostic. The origin of slight deviations between XICS and Thomson $T_{e}$ profiles in the order of $\Delta T_{e}<0.1-0.3 \mathrm{keV}$ in the region of $\rho=0.4-0.7$ is presently unknown but might be caused by uncertainties in the diagnostics viewing geometries or inaccuracies in the atomic data models used for both diagnostics. A cross comparison of experimentally obtained $T_{i}$ and impurity density profiles with a second imaging spectrometer installed at W7-X can be found in 1 .

\section{CONCLUSION}

In this work, an inversion method for the inference of temperature $\left(T_{i}\right.$ and $\left.T_{e}\right)$ and impurity density profiles out of line integrated data provided by X-ray imaging crystal spectrometers has been presented for the example of the XICS diagnostic.

On the basis of an entire forward model of the diagnostic, measured data have been synthesized for assumed temperature and density profiles. The inference is realized by a variation of free model parameters with respect to an optimal fit between synthesized and forward modeled data.

Results show a reliable inference of radial plasma parameter profiles with no more than $2 \sigma$ deviations from the synthesized data. Applied to measured data, obtained $T_{e}$ profiles match reasonably good to local measurements of 
the Thomson scattering diagnostic with maximum deviations of $\Delta T_{e}<0.3 \mathrm{keV}$.

\section{ACKNOWLEDGMENTS}

For various discussions, the authors would like to thank Jonathan Schilling, Udo Höfel, Andrea Pavone, and Andreas Dinklage.

This work has been carried out within the framework of the EUROfusion Consortium and has received funding from the Euratom research and training programme 2014-2018 and 2019-2020 under grant agreement No 633053. The views and opinions expressed herein do not necessarily reflect those of the European Commission.

${ }^{1}$ A. Langenberg, N. A. Pablant, T. Wegner, P. Traverso, O. Marchuk, T. Bräuer, B. Geiger, G. Fuchert, S. Bozhenkov, E. Pasch, O. Grulke, F. Kunkel, C. Killer, D. Nicolai, G. Satheeswaran, K. P. Hollfeld, B. Schweer, T. Krings, P. Drews, G. Offermanns, A. Pavone, J. Svensson, J. A. Alonso, R. Burhenn, and R. C. Wolf, Review of Scientific Instruments 89, 10G101 (2018) https://doi.org/10.1063/1.5036536.

${ }^{2}$ M. Krychowiak, A. Adnan, A. Alonso, T. Andreeva, J. Baldzuhn, T. Barbui, M. Beurskens, W. Biel, C. Biedermann, B. D. Blackwell, H. S. Bosch, S. Bozhenkov, R. Brakel, T. Bräuer, B. B. de Carvalho, R. Burhenn, B. Buttenschön, A. Cappa, G. Cseh, A. Czarnecka, A. Dinklage, P. Drews, A. Dzikowicka, F. Effenberg, M. Endler, V. Erckmann, T. Estrada, O. Ford, T. Fornal, H. Frerichs, G. Fuchert, J. Geiger, O. Grulke, J. H. Harris, H. J. Hartfuß, D. Hartmann, D. Hathiramani, M. Hirsch, U. Höfel, S. Jablonski, M. W. Jakubowski, J. Kaczmarczyk, T. Klinger, S. Klose, J. Knauer, G. Kocsis, R. König, P. Kornejew, A. Kramer-Flecken, N. Krawczyk, T. Kremeyer, I. Ksiazek, M. Kubkowska, A. Langenberg, H. P. Laqua, M. Laux, S. Lazerson, Y. Liang, S. C. Liu, A. Lorenz, A. O. Marchuk, S. Marsen, V. Moncada, D. Naujoks, H. Neilson, O. Neubauer, U. Neuner, H. Niemann, J. W. Oosterbeek, M. Otte, N. Pablant, E. Pasch, T. S. Pedersen, F. Pisano, K. Rahbarnia, L. Ryc, O. Schmitz, S. Schmuck, W. Schneider, T. Schröder, H. Schuhmacher, B. Schweer, B. Standley, T. Stange, L. Stephey, J. Svensson, T. Szabolics, T. Szepesi, H. Thomsen, J.-M. Travere, H. T. Mora, H. Tsuchiya, G. M. Weir, U. Wenzel, A. Werner, B. Wiegel, T. Windisch, R. Wolf, G. A. Wurden, D. Zhang, A. Zimbal, and S. Zoletnik, Review of Scientific Instruments 87, 11D304 (2016) http://aip.scitation.org/doi/pdf/10.1063/1.4964376

${ }^{3}$ H. Thomsen, A. Langenberg, D. Zhang, G. Bertschinger, C. Biedermann, W. Biel, R. Burhenn, B. Buttenschön, K. Grosser, R. König, M. Kubkowska, O. Marchuk, N. Pablant, L. Ryc, T. Pedersen, and W.-X. team, Journal of Instrumentation 10, P10015 (2015)

${ }^{4}$ N. A. Pablant, M. Bitter, R. Burhenn, L. Delgado-Aparicio, R. Ellis, D. Gates, M. Goto, K. W. Hill, A. Langenberg, S. Lazerson, M. Mardenfeld, S. Morita, G. H. Neilson, and T. Oishi, in 41st EPS conference, ECA, Vol. 38F (2014) p. 1.076.

${ }^{5}$ H. H. Johann, Zeitschrift für Physik 69, 185 (1931)

${ }^{6}$ H. Chen, M. Bitter, K. W. Hill, S. Kerr, E. Magee, S. R. Nagel, J. Park, M. B. Schneider, G. Stone, G. J. Williams, and P. Beiersdorfer, Review of Scientific Instruments 85, 11E606 (2014)

'N. A. Pablant, M. Bitter, L. Delgado-Aparicio, M. Goto, K. W. Hill, S. Lazerson, S. Morita, A. L. Roquemore, D. Gates, D. Monticello, H. Nielson, A. Reiman, M. Reinke, J. E. Rice, and H. Yamada, Review of Scientific Instruments 83, 083506 (2012)

${ }^{8}$ F. Wang, Y. Shi, W. Zhang, J. Fu, Y. Li, J. Xu, Y. Shen, B. Lu, J. Zhang, B. Wan, S. Lee, M. Bitter, and K. Hill, Journal of the Korean Physical Society 59, 2734 (2011)
${ }^{9}$ K. W. Hill, M. Bitter, L. Delgado-Aparicio, D. Johnson, R. Feder, P. Beiersdorfer, J. Dunn, K. Morris, E. Wang, M. Reinke, Y. Podpaly, J. E. Rice, R. Barnsley, M. O'Mullane, and S. G. Lee, Review of Scientific Instruments 81, 10E322 (2010)

${ }^{10} \mathrm{G}$. Bertschinger and O. Marchuk, Burning Plasma Diagnostics 988, 189 (2008).

${ }^{11}$ G. Bertschinger, W. Biel, H. Jaegers, and O. Marchuk, Review of Scientific Instruments 75, 3727 (2004).

${ }^{12}$ J. E. Rice, J. L. Terry, E. S. Marmar, R. S. Granetz, M. J. Greenwald, A. E. Hubbard, J. H. Irby, S. M. Wolfe, and T. S. Pedersen, Fusion Science and Technology 51, 357 (2007).

${ }^{13}$ O. Marchuk, M. Z. Tokar, G. Bertschinger, A. Urnov, H.J. Kunze, D. Pilipenko, X. Loozen, D. Kalupin, D. Reiter, A. Pospieszczyk, W. Biel, M. Goto, and F. Goryaev, Plasma Physics and Controlled Fusion 48, 1633 (2006)

${ }^{14}$ A. Langenberg, J. Svensson, H. Thomsen, O. Marchuk, N. A. Pablant, R. Burhenn, and R. C. Wolf, Fusion Science and Technology 69, 560 (2016) http://dx.doi.org/10.13182/FST15-181

${ }^{15}$ A. Ince-Cushman, J. E. Rice, M. Bitter, M. L. Reinke, K. W. Hill, M. F. Gu, E. Eikenberry, C. Broennimann, S. Scott, Y. Podpaly, S. G. Lee, and E. S. Marmar, Review of Scientific Instruments 79, 10E302 (2008)

${ }^{10} \mathrm{M}$. El Fagrich and H. Chehouani, Optics and Lasers In Engineering 50, $336(2012)$

${ }^{17}$ S. Ma, H. Gao, L. Wu, and G. Zhang, Journal of Quantitative Spectroscopy \& Radiative Transfer 109, 1745 (2008)

${ }^{18}$ G. Fussmann, H. Meyer, and E. Pasch, Contributions To Plasma Physics 36, 501 (1996)

${ }^{19}$ L. M. Smith and D. R. Keefer, Journal of Quantitative Spectroscopy \& Radiative Transfer 39, 367 (1988).

${ }^{20}$ A. Langenberg, H. Thomsen, R. Burhenn, O. Marchuk, J. Svensson, T. S. Pedersen, and R. C. Wolf, in 41st EPS conference, $E C A$, Vol. $38 \mathrm{~F}$ (2014) p. 1.074.

${ }^{21}$ N. A. Pablant, R. E. Bell, M. Bitter, L. Delgado-Aparicio, K. W. Hill, S. Lazerson, and S. Morita, Review of Scientific Instruments 85, 11E424 (2014)

${ }^{22}$ D. Li, J. Svensson, H. Thomsen, F. Medina, A. Werner, and R. C. Wolf, Review of Scientific Instruments 84, 083506 (2013)

${ }^{23}$ M. L. Reinke, Y. A. Podpaly, M. Bitter, I. H. Hutchinson, J. E. Rice, L. Delgado-Aparicio, C. Gao, M. Greenwald, K. Hill, N. T. Howard, A. Hubbard, J. W. Hughes, N. Pablant, A. E. White, and S. M. Wolfe, Review of Scientific Instruments 83, 113504 (2012) http://dx.doi.org/10.1063/1.4758281

${ }^{24}$ J. Svensson and A. Werner, Plasma Physics and Controlled Fusion 50, $085002(2008)$

${ }^{25}$ L. C. Ingesson, H. Chen, P. Helander, and M. J. Mantsinen, Plasma Physics and Controlled Fusion 42, 161 (2000)

${ }^{26}$ I. Condrea, E. Haddad, B. C. Gregory, and G. Abel, Physics of Plasmas 7, 3641 (2000)

${ }^{2 / R}$ R. E. Bell, Review of Scientific Instruments 68, 1273 (1997)

${ }^{28}$ J. Svensson and A. Werner, in International Symposium on Intelligent Signal Processing-WISP (2007) pp. 955-960.

${ }^{29}$ U. Höfel, S. Bozhenkov, and G. Fuchert, in 43rd European Physical Society Conf. on Plasma Physics (Leuven, Belgium)|(2016) p. 4.008 .

${ }^{30}$ S. Bozhenkov, M. Beurskens, A. D. Molin, G. Fuchert, E. Pasch, M. Stoneking, M. Hirsch, U. Höfel, J. Knauer, J. Svensson, H. T. Mora, and R. Wolf, Journal of Instrumentation 12, P10004 (2017)

${ }^{3}$ S. K. Rathgeber, L. Barrera, T. Eich, R. Fischer, B. Nold, W. Suttrop, M. Willensdorfer, and E. Wolfrum, Plasma Physics and Controlled Fusion 55, 025004 (2013)

${ }^{32}$ O. Ford, Tokamak Plasma Analysis through Bayesian Diagnostic Modelling, Ph.D. thesis, University of London, Imperial College London (2010).

${ }^{33}$ J. Svensson, A. Dinklage, J. Geiger, A. Werner, and R. Fischer, Review of Scientific Instruments 75, 4219 (2004)

${ }^{34}$ M. Krychowiak, R. Konig, T. Klinger, and R. Fischer, Journal of Applied Physics 96, 4784 (2004) 
${ }^{35}$ R. Fischer, A. Dinklage, and E. Pasch, Plasma Physics and Controlled Fusion 45, 1095 (2003).

${ }^{36} \mathrm{~J}$. Svensson and R. König, in 32nd EPS Conference on Plasma Physics (2005) p. 5.087.

${ }^{37} \mathrm{~J}$. Svensson, "Non-parametric tomography using gaussian processes," Tech. Rep. (JET internal report, EFDA-JET-PR(11)24, 2010).

${ }^{38}$ M. A. Chilenski, M. Greenwald, Y. Marzouk, N. T. Howard, A. E. White, J. E. Rice, and J. R. Walk, Nuclear Fusion 55, 023012 (2015)

${ }^{39}$ J. Ashburner and K. J. Friston, Human Brain Mapping 7, 254 (1999).

${ }^{40}$ J. C. Carr, W. R. Fright, and R. K. Beatson, IEEE Transactions On Medical Imaging 16, 96 (1997)

${ }^{41}$ R. Preuss and U. von Toussaint, Entropy 20, 201 (2018)

${ }^{42}$ M. Drevlak, D. Monticello, and A. Reiman, Nuclear Fusion 45, $731(2005)$

${ }^{43} \mathrm{O}$. Marchuk, Modeling of He-like spectra measured at the tokamaks TEXTOR and TORE SUPRA, Ph.D. thesis, Ruhr Universität Bochum (2004).

${ }^{44}$ O. Marchuk, G. Bertschinger, H.-J. Kunze, A. Urnov, and F. Goryaev, Journal of Physics B - Atomic Molecular and Optical Physics 40, 4403 (2007)

${ }^{45}$ M. Bitter, M. F. Gu, L. A. Vainshtein, P. Beiersdorfer, G. Bertschinger, O. Marchuk, R. Bell, B. LeBlanc, K. W. Hill, D. Johnson, and L. Roquemore, Phys. Rev. Lett. 91, 265001 (2003).

${ }^{40} \mathrm{~F}$. Goryaev, L. Vainshtein, and A. Urnov, Atomic Data and Nuclear Data Tables 113, 117 (2017)

${ }^{4}$ Bautista, M. A. and Badnell, N. R., A\&A 466, 755 (2007)

${ }^{48}$ M. F. Gu, The Astrophysical Journal 590, 1131 (2003)

${ }^{49}$ R. Wolf, A. Ali, A. Alonso, J. Baldzuhn, C. Beidler, M. Beurskens, C. Biedermann, H.-S. Bosch, S. Bozhenkov, and R. Brakel, Nuclear Fusion 57, 102020 (2017)

${ }^{50}$ S. P. Brooks, Journal of the Royal Statistical Society Series D The Statistician 47, 69 (1998).

${ }^{51}$ S. Chib and E. Greenberg, American Statistician 49, 327 (1995)

${ }^{52}$ J. Besag, P. Green, D. Higdon, and K. Mengersen, Statistical Science 10, 3 (1995)

${ }^{53}$ R. V. Craiu and J. S. Rosenthal, Annual Review of Statistics and Its Application 1, 179 (2014)

${ }^{54}$ T. Pedersen, A. Dinklage, Y. Turkin, R. Wolf, S. Bozhenkov, J. Geiger, G. Fuchert, H. S. Bosch, K. Rahbarnia, H. Thomsen, U. Neuner, T. Klinger, A. Langenberg, H. T. Mora, P. Kornejew, J. Knauer, M. Hirsch, and N. Pablant, Physics of Plasmas 24,
055503 (2017) http://dx.doi.org/10.1063/1.4983629

${ }^{5}$ R. Dux, STRA HL user manual, Max-Planck-Institue for Plasmaphysics (2006), IPP Report 10/30.

${ }^{56}$ A. Langenberg, N. Pablant, O. Marchuk, D. Zhang, J. Alonso, R. Burhenn, J. Svensson, P. Valson, D. Gates, M. Beurskens, R. Wolf, and the W7-X Team, Nuclear Fusion 57, 086013 (2017).

${ }^{57}$ H. P. Summers and M. G. O'Mullane, AIP Conference Proceedings 1344, 179 (2011)

${ }^{5 \gamma}$ Y. Feng, H. Frerichs, M. Kobayashi, A. Bader, F. Effenberg, D. Harting, H. Hoelbe, J. Huang, G. Kawamura, J. D. Lore, T. Lunt, D. Reiter, O. Schmitz, and D. Sharma, Contributions to Plasma Physics 54, 426 (2014)

${ }^{59}$ H. Maassberg, C. D. Beidler, and E. E. Simmet, Plasma Physics and Controlled Fusion 41, 1135 (1999)

${ }^{\mathrm{ou}}$ N. A. Pablant, A. Langenberg, A. Alonso, C. D. Beidler, M. Bitter, S. Bozhenkov, R. Burhenn, M. Beurskens, L. DelgadoAparicio, A. Dinklage, G. Fuchert, D. Gates, J. Geiger, K. W. Hill, U. Höfel, M. Hirsch, J. Knauer, A. Krämer-Flecken, M. Landreman, S. Lazerson, H. Maaßberg, O. Marchuk, S. Massidda, G. H. Neilson, E. Pasch, S. Satake, J. Svennson, P. Traverso, Y. Turkin, P. Valson, J. L. Velasco, G. Weir, T. Windisch, R. C. Wolf, M. Yokoyama, D. Zhang, and the W7-X Team, Physics of Plasmas 25, 022508 (2018) https://doi.org/10.1063/1.4999842

${ }^{01}$ J. Arevalo, J. A. Alonso, K. J. McCarthy, J. L. Velasco, J. M. Garcia-Regana, and M. Landreman, Nuclear Fusion 54, 013008 (2014)

${ }^{\overline{6} 2}$ J. Arevalo, J. A. Alonso, K. J. McCarthy, and J. L. Velasco, Nuclear Fusion 53, 023003 (2013)

${ }^{63}$ J. M. Garcia-Regana, R. Kleiber, C. D. Beidler, Y. Turkin, H. Maassberg, and P. Helander, Plasma Physics and Controlled Fusion 55, 074008 (2013)

${ }^{64}$ J. Rice, J. Terry, E. Marmar, and F. Bombarda, Nuclear Fusion 37, 241 (1997)

${ }^{65}$ J. Garcia-Regana, C. Beidler, R. Kleiber, P. Helander, A. Mollen, J. Alonso, M. Landreman, H. Maaßberg, H. Smith, Y. Turkin, and J. Velasco, Nuclear Fusion 57, 056004 (2017)

${ }^{66}$ A. Langenberg, F. Warmer, G. Fuchert, O. Marchuk, A. Dinklage, T. Wegner, J. A. Alonso, S. Bozhenkov, K. J. Brunner, R. Burhenn, B. Buttenschön, P. Drews, B. Geiger, O. Grulke, M. Hirsch, U. Höfel, K. P. Hollfeld, C. Killer, J. Knauer, T. Krings, F. Kunkel, U. Neuner, G. Offermanns, N. A. Pablant, E. Pasch, K. Rahbarnia, G. Satheeswaran, J. Schilling, B. Schweer, H. Thomsen, P. Traverso, R. C. Wolf, and the W7-X Team, Plasma Physics and Controlled Fusion 61, 014030 (2019) 